\title{
Prevalência e correlatos do uso problemático de smartphones por graduandos da área da saúde
}

\author{
Prevalence and correlates of problematic smartphone use \\ in undergraduate students on health courses
}

Prevalencia y correlaciones del uso problemático de teléfonos inteligentes por parte de estudiantes universitarios en salud

Gabriel Acácio Pena de Menezes - https://orcid.org/0000-0003-4440-6793 João Pedro Paz Takeuchi - https://orcid.org/0000-0001-6276-9585 Luciana Alves Silveira Monteiro - https://orcid.org/0000-0002-1071-9946 Jonas Jardim de Paula - https://orcid.org/0000-0001-5530-2346

\section{Resumo}

Introdução: Os smartphones são considerados um gadget fundamental entre os jovens porque oferecem uma infinidade de serviços e operam muitas funções diferentes, acabando por substituir vários dispositivos. 0 uso problemático de smartphones tem implicações físicas e psicológicas. Objetivo: Encontrar a prevalência de uso problemático de smartphone (UPS) entre alunos de graduação de uma faculdade particular de saúde, em Belo Horizonte, MG, Brasil, e correlacionar esse comportamento com transtornos de humor, sono, falhas cognitivas, déficit de atenção e hiperatividade. Método: A amostra foi avaliada por meio da Escala Hospitalar de Ansiedade e Depressão (HADS), Escala de Sonolência de Epworth, Inventário de Dependência de Smartphone, Escala de Autorrelato de Adultos (ASRS-18) e Questionário de Falhas Cognitivas. Resultados: 156 voluntários responderam à pesquisa, a maioria estudantes de medicina. A prevalência de PSU foi de 52,5\%; que foi estaticamente correlacionado com sintomas de déficit de atenção e hiperatividade, 
transtornos de humor e transtornos do sono. A prevalência de sintomas de depressão e ansiedade foi de $66,6 \%$ e $82,69 \%$, respectivamente.

Conclusão: Concluímos que o PSU é um fenômeno prevalente entre estudantes da área de saúde, podendo estar associado a sonolência, transtornos do humor e sintomas de Déficit de Atenção e Hiperatividade.

Palavras-chave: Saúde Mental; Smartphone; Comportamento Aditivo; Transtorno de humor; Déficit de Atenção e Hiperatividade

\section{Abstract}

Introduction: Young people consider smartphones as essential because they offer an infinite range of services and operate many different functions, enabling them to substitute many different devices. Problematic smartphone use has both physical and psychological implications. Objective: To establish the prevalence of problematic smartphone use (PSU) among undergraduate students at a private medical school in Belo Horizonte, MG, Brazil, and to correlate this behavior with mood disorders, sleep disorders, cognitive disorders, and attention deficit hyperactivity disorder. Method: The sample was assessed using the Hospital Anxiety and Depression Scale (HADS), the Epworth Somnolence Scale, the Smartphone Dependence Inventory, the Adult Self-Report Scale (ASRS18), and the Cognitive Failure Questionnaire. Results: A total of 156 volunteers participated in the study, the majority of whom were medical students. The prevalence of PSU was $52.5 \%$ and was statistically correlated with symptoms of attention deficit and hyperactivity, mood disorders, and sleep disorders. The prevalence rates of symptoms of depression and anxiety were 66.6 and $82.69 \%$, respectively. Conclusions: We conclude that PSU is a prevalent phenomenon among students studying medicine and related areas and can be associated with somnolence, affective disorders, and symptoms of attention deficit and hyperactivity.

Keywords: Mental health, smartphone, addictive behavior, mood disorder, attention deficit, hyperactivity

\section{RESUMEN}

Introducción: Los jóvenes consideran que los teléfonos inteligentes son esenciales porque ofrecen una gama infinita de servicios y operan muchas funciones diferentes, lo que les permite sustituir muchos dispositivos 
Menezes, G.A.P; Takeuchi, J.P.P.; Monteiro, L.A.S.; Paula, J.J.

diferentes. El uso problemático de teléfonos inteligentes tiene implicaciones tanto físicas como psicológicas. Objetivo: Establecer la prevalencia del uso problemático de teléfonos inteligentes (PSU) entre estudiantes de pregrado de una escuela de medicina privada en Belo Horizonte, MG, Brasil, y correlacionar este comportamiento con trastornos del estado de ánimo, trastornos del sueño, trastornos cognitivos y trastorno por déficit de atención con hiperactividad. Método: La muestra se evaluó mediante la Escala Hospitalaria de Ansiedad y Depresión (HADS), la Escala de Somnolencia de Epworth, el Inventario de Dependencia de Smartphone, la Escala de Autoinforme de Adultos (ASRS-18) y el Cuestionario de Fallas Cognitivas. Resultados: Participaron en el estudio 156 voluntarios, la mayoría estudiantes de medicina. La prevalencia de PSU fue $52,5 \%$ y se correlacionó estadísticamente con síntomas de déficit de atención e hiperactividad, trastornos del estado de ánimo y trastornos del sueño. Las tasas de prevalencia de síntomas de depresión y ansiedad fueron 66,6 y $82,69 \%$, respectivamente. Conclusiones: Se concluye que la UPM es un fenómeno prevalente entre los estudiantes de medicina y áreas afines y puede estar asociado con somnolencia, trastornos afectivos y síntomas de déficit de atención e hiperactividad.

Palabras clave: salud mental, smartphone, comportamiento adictivo, trastorno del estado de ánimo, déficit de atención, hiperactividad

Como citar: Menezes, G.A.P; Takeuchi, J.P.P.; Monteiro, L.A.S.; Paula, J.J. Prevalência e correlatos do uso problemático de smartphones por graduandos da área da saúde. Debates em Psiquiatria, Rio de Janeiro, 2021; 11:1-13 https://doi.org/10.25118/2763-9037.2021.v11.56

Conflito de interesses: declaram não haver

Fonte de financiamento: declaram não haver

Parecer CEP: UFMG - CAAE no 20819119.0.0000.5134

Recebido em: 31/05/2021

Aprovado em: 31/05/2021

Publicado em: 13/09/2021 


\section{Introdução}

Smartphones são aparelhos considerados fundamentais pela maioria dos jovens, já que oferecem amplo acesso a serviços e podem desempenhar inúmeras funções, de maneira a dispensar o uso de múltiplos aparelhos em prol do uso de apenas um. Cerca de $40 \%$ dos jovens consideram as informações veiculadas por meio de smartphones significativas [1] , e 60\% apresentam comportamento de risco e susceptibilidade à dependência, especialmente aqueles que possuem acesso à internet em seus dispositivos móveis [2]].

Existem observações na literatura de que $40 \%$ dos graduandos e pósgraduandos apresentam comportamento de risco para dependência de smartphones, especialmente entre mulheres que se queixam de solidão e tédio. Apesar da prevalência alarmante, é também relatado que apenas $1 \%$ dos usuários de smartphones podem ser considerados altamente prejudicados [ $\underline{3}]$. Esse comportamento aditivo está relacionado, mas não apenas, à história parental de uso patológico de smartphones, isolamento social, redução da capacidade de autocontrole, transtornos de humor e fatores socioculturais [ㅁ-ㅁ] . Nessa mesma linha de pensamento, a literatura também sugere que boas relações entre pares não podem ser consideradas fator protetor para o uso problemático de smartphones (UPS) [7]. Apesar disso, inexiste um modelo psicopatológico adequado para explicar o UPS.

Existe associação entre UPS e insônia em adolescentes e jovens adultos []․ UPS também pode ser correlacionado com problemas osteomusculares e falhas cognitivas, inclusive promotoras de situações de risco contra a vida em casos de déficit de atenção, que podem implicar em acidentes de tráfego [9-12]. Em somatória, estudos anteriores estabeleceram correlações fortes entre UPS com somatização, transtornos de humor e comportamento disruptivo entre adolescentes em tratamento psiquiátrico [13].

Este estudo visa investigar a prevalência do UPS entre graduandos de uma instituição de ensino superior privada da área da saúde (que oferece cursos de enfermagem, fisioterapia, medicina e psicologia) de Belo Horizonte $(\mathrm{MG})$, Brasil. Além disso, pesquisaram-se possíveis associações de depressão e ansiedade, dado que esses transtornos são prevalentes tanto em profissionais [14] quanto em estudantes da área da saúde $[\underline{15}, \underline{16}]$. 
Menezes, G.A.P; Takeuchi, J.P.P.; Monteiro, L.A.S.; Paula, J.J.

\section{Métodos}

Este estudo foi aprovado pelo Comitê de Ética em Pesquisa da instituição na qual foi conduzido (CAAE no 20819119.0.0000.5134). Trata-se de um estudo transversal observacional de amostra não probabilística. Os voluntários (todos com 18 anos ou mais) receberam um questionário online com seis seções, ao qual deveriam responder anonimamente. Por questões éticas, cada voluntário forneceu eletronicamente seu consentimento livre e esclarecido antes da coleta dos dados.

$\mathrm{Na}$ primeira seção, os voluntários deveriam informar o curso em que estavam matriculados e qual ano/período estavam cursando. O restante do questionário era composto por instrumentos psicométricos, a saber: Hospital Anxiety and Depression Scale (HADS), analisando-se isoladamente seu componente depressivo (D) e o ansioso (A) [17]; Escala de Sonolência de Epworth (ESS) [18], para que fosse possível inferir transtornos relacionados ao sono; Smartphone Addiction Inventory (SPAI) [19]; Adult Self Report Scale (ASRS-18) [20], adotado como um instrumento para quantificar sintomas do transtorno de déficit de atenção e hiperatividade (TDAH); e Questionário de Falhas Cognitivas [21] (CFQ). Esses instrumentos foram aplicados em suas versões devidamente traduzidas e adaptadas culturalmente ao público brasileiro.

Como a maioria dos estudantes matriculados na instituição em questão está cursando medicina (aproximadamente $80 \%$ dos participantes), optouse por agrupar os voluntários em grupos, separando estes daqueles que cursam os demais programas de graduação; assim, para analisar os dados coletados, é válido mencionar que um grupo era composto por estudantes que possuíam dedicação em tempo integral ao curso (medicina) e o outro por alunos com dedicação em um turno (enfermagem, fisioterapia e psicologia). A análise estatística foi feita por meio do programa JASP (versão 0.11.1; ASP Team, 2019). A associação entre as variáveis foi verificada por meio do coeficiente de correlação de Spearman.

\section{Resultados}

Ao todo, 156 estudantes aceitaram participar como voluntários desta pesquisa. Destes, 116 eram graduandos de medicina e 40 eram graduandos dos outros cursos da instituição (18 de psicologia, 14 de enfermagem e oito de fisioterapia). Nesta amostra, 13 estudantes estavam matriculados no primeiro semestre, 24 no segundo, 37 no terceiro, 20 no 
quarto, 30 no quinto, cinco no sexo, 14 no sétimo, seis no oitavo, seis no nono e um no décimo.

Acerca da dependência de smartphone, 52,5\% da amostra preenchia critérios suficientes para qualificar o UPS segundo o SPAI (adotando ponto de corte de 10). Além disso, $66 \%$ dos voluntários preenchiam critérios para possível diagnóstico de depressão (ponto de corte de 5) e ainda mais alunos $(82,69 \%)$ para ansiedade (ponto de corte de 6 ).

Foi verificada também correlação positiva e estatisticamente significante entre UPS e sintomas atencionais e de hiperatividade, ansiedade, depressão e sonolência. Não foi possível verificar correlação entre USP e falhas cognitivas. Os resultados podem ser verificados na Tabela 1.

Tabela 1: Correlações de Spearman entre uso problemático de smartphones, mensurado por meio do Smartphone Addiction Inventory, e variáveis referentes à saúde mental

Correlação com a SPAI

Questionário de Falhas Cognitivas

HADS (Ansiedade)

HADS (Depressão)

Escala de Sonolência de Epworth

ASRS-18 Hiperatividade

ASRS-18

\section{(rho)}

0.018

$0.251^{*}$

$0.243^{*}$

$0.232 *$

$0.339+$

$0.437+$

HADS: Hospital Anxiety and Depression Scale, SPAI: Smartphone Addiction Inventory, ASRS-18: Adult Self Report Scale. $* p<0.05,+p<0.01$

\section{Discussão}

Cerca de metade da amostra estudada (52,5\%) apresentou uso elevado e disfuncional de smartphones, que pode ser caracterizado pelo SPAI como uma dependência. Esse valor é significativamente maior do que o 
Menezes, G.A.P; Takeuchi, J.P.P.; Monteiro, L.A.S.; Paula, J.J.

encontrado em estudos populacionais (10-30\%) [22], apesar de corroborar com o achado de outro estudo conduzido em Belo Horizonte

com universitários de outra instituição [23]. Uma possível explicação para tais achados é que smartphones são utilizados como um instrumento de coping, já que se sabe que os níveis de estresse entre estudantes da área da saúde são maiores que os da população geral [24].

Os achados desta pesquisa também indicam correlação entre UPS e ansiedade e depressão, o que pode ser, na percepção dos autores, entendido de duas maneiras: pode-se entender que ansiedade e depressão são fatores de risco para o desenvolvimento de UPS [25-26]; outra possibilidade é que esses transtornos, na verdade, são consequência do UPS [26]. Como ambas as possibilidades são plausíveis, estudos devidamente desenhados para avaliar essa questão são necessários.

Especificamente sobre a correlação entre UPS e ansiedade, os achados corroboram aqueles encontrados na literatura internacional [27]. Isso é verificado nos casos dos sintomas de hiperatividade e déficit de atenção [28-29]. Ressaltamos que, como a impulsividade é um sintoma importante do TDAH e é um fator relevante na psicopatologia das dependências, esses achados eram esperados [30].

Por fim, foi encontrada uma correlação significativa entre PSU e sonolência diurna, confirmando os achados de estudos feitos em outras comunidades $[\underline{22}, \underline{31}]$. Tal relação pode ser explicada pela prolongada exposição à luz à noite, afetando o ciclo circadiano e gerando sonolência. Os pesquisadores gostariam de ressaltar a emergência dessa área de pesquisa recentemente e a oferta de inovações tecnológicas que podem mitigar esta problemática [32].

Considerando a única variável que não foi possível correlacionar estatisticamente com UPS (falhas cognitivas), observa-se discordância com estudos prévios em que esta foi verificada [33] , ainda que a evidência não seja robusta. Nossos achados sugerem que voluntários com UPS não desenvolvem clinicamente manifestações cognitivas; isso apoia o entendimento de UPS como uma condição desadaptativa, e não um transtorno primário.

É importante observar que esta pesquisa possui limitações. A amostra não foi representativa, além de ter envolvido poucos alunos no fim da graduação e poucos alunos de cursos que não são de período integral.

7 Debates em Psiquiatria, Rio de Janeiro, 2021, 11:1-13 
Em somatória, apesar de os instrumentos em questão serem adequados para a população em estudo, eles valem-se de autorrelatos, que não necessariamente refletem os reais comportamentos dos voluntários.

É também relevante mencionar que os dados foram coletados antes da pandemia de COVID-19, então os achados deste trabalho não podem ser extrapolados para o contexto de crise sanitária, o que requer estudos especialmente desenhados para tal situação.

\section{Conclusão}

Conclui-se que UPS é um fenômeno prevalente entre estudantes da área da saúde e pode ser associado com sonolência, depressão, ansiedade e sintomas de TDAH. Esses resultados reforçam a necessidade de mais pesquisas para entender os prejuízos causados pelo UPS e suas repercussões na saúde mental.

\section{Agradecimentos}

Este trabalho foi apoiado pela Ciências Médicas de Minas Gerais, por meio do Programa de Bolsas de Iniciação Científica Institucional (PROBIC) 2\%/2019 e pela Liga Acadêmica de Saúde Mental CMMG, um grupo de estudos do qual todos os autores deste trabalho eram membros durante a execução desta pesquisa. 
Menezes, G.A.P; Takeuchi, J.P.P.; Monteiro, L.A.S.; Paula, J.J.

\section{Referências}

1. Gutiérrez-Rentería ME, Santana-Villegas JC, Pérez-Ayala M. Smartphone: usos y gratificaciones de los jóvenes en México en 2015. Palabra Clave. 2015;20:47-68. https://doi.org/10.5294/pacla.2017.20.1.3

- 2. Medrano JL, Rosales FL, Loving RD. Conducta adictiva a las redes sociales y su relación con el uso problemático del móvil. Acta Investig Psicol. 2017; 7:2832-38.

https://doi.org/10.1016/j.aipprr.2017.11.001

3. Ruiz-Palmero J, Sánchez-Rodríguez J, Trujillo Torres JM. Utilización de Internet y dependencia a teléfonos móviles en adolescentes. Rev Latinoam Cienc Soc Niñez Juv. 2016;14:1357-69.

- 4. Borges AP, Joia LA. Executivos e smartphones: uma relação ambígua e paradoxal. Organ Soc. 2013;20:585-602. https://doi.org/10.1590/S1984-92302013000400002

5. Youn H, Lee SI, Lee SH, Kim JY, Kim JH, Park EJ, et al. Exploring the differences between adolescents' and parents' ratings on adolescents' smartphone addiction. J Korean Med Sci.

2018;33:e347. https://doi.org/10.3346/jkms.2018.33.e347 PMid:30584419 PMCid:PMC6300655

6. Lee EJ, Kim HS. Gender differences in smartphone addiction behaviors associated with parent-child bonding, parent-child communication, and parental mediation among Korean elementar school students. J Addict Nurs. 2018;29:244-54.

7. Ihm J. Social implications of children's smartphone addiction: the role of support networks and social engagement. J Behav Addict. 2018;7:473-81. https://doi.org/10.1556/2006.7.2018.48 PMid:29865865 PMCid:PMC6174576

- 8. Rod NH, Dissing AS, Clark A, Gerds TA, Lund R. Overnight smartphone use: a new public health challenge? A novel study design based on high-resolution smartphone data. PloS One. 2018; 13:e0204811.

9. Chen PL, Pai CW. Pedestrian smartphone overuse and inattentional blindness: an observational study in Taipei, Taiwan. 
BMC Public Health. 2018;18:1342. https://doi.org/10.1186/s12889018-6163-5 PMid:30595132 PMCid:PMC6311895

10. Fowler J, Noyes J. A study of the health implications of mobile phone use in 8-14s. Dyna (Medellin, Colombia). 2017;84:228-33. https://doi.org/10.15446/dyna.v84n200.62156

11. Namwongsa S, Puntumetakul R, Neubert MS, Boucaut R. Factors associated with neck disorders among university student smartphone users. Work. 2018;61:367-78.

https://doi.org/10.3233/WOR-182819 PMid:30373996

12. Queiroz LB, Lourenço B, Silva LE, Lourenço DM, Silva CA. Dor musculoesquelética e síndromes musculoesqueléticas em adolescentes relacionadas a dispositivos eletrônicos. J Pediatr (Rio J). 2018;94:673-9. https://doi.org/10.1136/postgradmedj-2018136145 PMid: 30554170

늘 13. Fırat S, Gül H, Sertçelik M, Gül A, Gürel Y, Kılıç BG. The relationship between problematic smartphone use and psychiatric symptoms among adolescents who applied to psychiatry clinics. Psychiatry Res. 18;270:97-103. https://doi.org/10.1016/j.psychres.2018.09.015 PMid:30245382

- 14. Knuth BS, da Silva RA, Oses JP, Radtke VA, Cocco RA, Jansen K. Mental disorders among health workers in Brazil. Cien Saude Colet. 2015;20:2481-8. https://doi.org/10.1590/1413-

81232015208.05062014 PMid:26221813

ㄴ 15. Rotenstein LS, Ramos MA, Torre M, Segal JB, Peluso MJ, Guille $\mathrm{C}$, et al. Prevalence of depression, depressive symptoms, and suicidal ideation among medical students: a systematic review and meta analysis. JAMA. 2016;316:2214-36.

https://doi.org/10.1001/jama.2016.17324 PMid:27923088 PMCid:PMC5613659

16. Tabalipa FD, de Souza MF, Pfützenreuter G, Lima VC, Traebert E, Traebert J. Prevalence of anxiety and depression among medical students. Rev. Bras. Educ. Med. 2015;39:388-94. https://doi.org/10.1590/1981-52712015v39n3e02662014

17. Faro A. Análise fatorial confirmatória e normatização da Hospital Anxiety and Depression Scale (HADS). Psic Teor Pesq. 2015;31:34953. https://doi.org/10.1590/0102-37722015032072349353 
Menezes, G.A.P; Takeuchi, J.P.P.; Monteiro, L.A.S.; Paula, J.J.

18. Bertolazi AN, Fagondes SC, Hoff LS, Pedro VD, Menna Barreto SS, Johns MW. Validação da escala de sonolência de Epworth em português para uso no Brasil. J Bras Pneumol. 2009;35:877-83. https://doi.org/10.1590/S1806-37132009000900009

PMid: 19820814

19. Khoury JM. Tradução, adaptação cultural e validação de uma versão brasileira do questionário Smartphone Addiction Inventory (SPAI) para o rastreamento de dependência de smartphone. (thesis). Belo Horizonte: Universidade Federal de Minas Gerais; 2016.

- 20. Mattos P, Segenreich D, Saboya E, Louzã M, Dias G, Romano M. Adaptação transcultural para o português da escala Adult SelfReport Scale para avaliação do transtorno de déficit de atenção/hiperatividade (TDAH) em adultos. Arch. Clin. Psychiatry (São Paulo). 2006;33:188-94. https://doi.org/10.1590/S010160832006000400004

21. de Paula JJ, Costa DS, Miranda DM, Romano-Silva MA. Brazilian version of the Cognitive Failures Questionnaire (CFQ): cross-cultural adaptation and evidence of validity and reliability.

Debates.2018;40:312-5.

22. Sohn SY, Rees P, Wildridge B, Kalk NJ, Carter B. Prevalence of problematic smartphone usage and associated mental health outcomes amongst children and young people: a systematic review, meta-analysis and GRADE of the evidence. BMC Psychiatry. 2019;19:356.

23. Khoury JM, das Neves MD, Roque MA, de Freitas AA, da Costa MR, Garcia FD. Smartphone and Facebook addictions share common risk and prognostic factors in a sample of undergraduate students. Trends Psychiatry Psychother. 2019;41:358-68. https://doi.org/10.1590/2237-6089-2018-0069 PMid:31967196

24. Jacob T, Itzchak EB, Raz O. Stress among healthcare students-a cross disciplinary perspective. Physiother Theory Pract.

2013;29:401-12. https://doi.org/10.3109/09593985.2012.734011 PMid:23094641 
- 25. Elhai JD, Tiamiyu M, Weeks J. Depression and social anxiety in relation to problematic smartphone use. Internet Res. 2018;28:31532. https://doi.org/10.1108/IntR-01-2017-0019

26. Extremera N, Quintana-Orts C, Sánchez Álvarez N, Rey L. The role of cognitive emotion regulation strategies on problematic smartphone use: comparison between problematic and non problematic adolescent users. Int J Environ Res Public Health. 2019;16:3142. https://doi.org/10.3390/ijerph16173142 PMid:31466410 PMCid:PMC6747355

27. Pinto NA, Cavestro JM, Fereira W. Prevalência de transtorno de ansiedade generalizada em estudantes de medicina. Rev Interdiscip Ciencias Med. 2018;2:36-43.

28. Carvalho LF, Sette CP, Ferrari BL. Problematic smartphone use relationship with pathological personality traits: systematic review and meta analysis. Cyberpsychology (Brno). 2018;12:Article 5. https://doi.org/10.5817/CP2018-3-5

29. Montagni I, Guichard E, Kurth T. Association of screen time with self-perceived attention problems and hyperactivity levels in French students: a cross-sectional study. BMJ Open. 2016;6:e009089.

30. Dey M, Studer J, Schaub MP, Gmel G, Ebert DD, Lee JY, et al. Problematic smartphone use in young Swiss men: Its association with problematic substance use and risk factors derived from the pathway model. J Behav Addict. 2019;8:326-34.

https://doi.org/10.1556/2006.8.2019.17 PMid:31079472

PMCid:PMC7044551

31. Kumar VA, Chandrasekaran V, Brahadeeswari H. Prevalence of smartphone addiction and its effects on sleep quality: a crosssectional study among medical students. Ind Psychiatry $\mathrm{J}$. 2019;28:82-5. https://doi.org/10.4103/ipj.ipj 5619 PMid:31879452 PMCid:PMC6929238

32. Oh JH, Yoo H, Park HK, Do YR. Analysis of circadian properties and healthy levels of blue light from smartphones at night. Sci Rep. 2015;5:11325. https://doi.org/10.1038/srep11325 PMid:26085126 PMCid:PMC4471664

33. Hadlington LJ. Cognitive failures in daily life: exploring the link with Internet addiction and problematic mobile phone use. Comput 
Menezes, G.A.P; Takeuchi, J.P.P.; Monteiro, L.A.S.; Paula, J.J.

Human Behav. 2015;51:75-81.

https://doi.org/10.1016/j.chb.2015.04.036

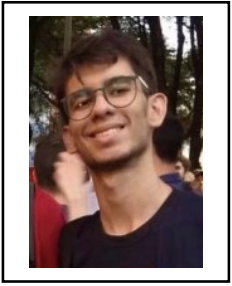

Gabriel Acácio Pena de Menezes

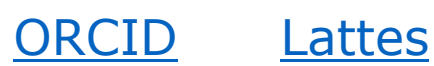

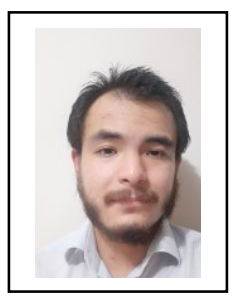

João Pedro Paz Takeuchi

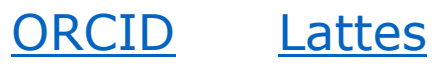

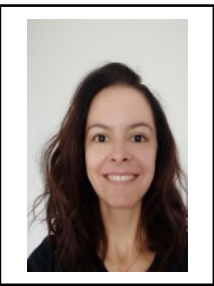

Luciana Alves Silveira Monteiro

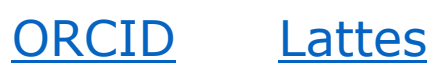

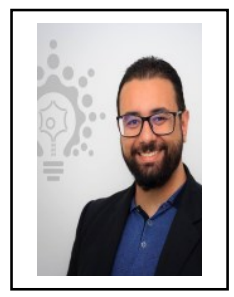

Jonas Jardim de Paula (autor correspondente)

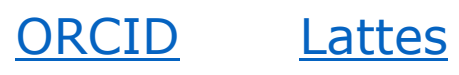

